EPiC Series in Engineering
Volume 3, 2018, Pages 1589-1597
HIC 2018. 13th International
Conference on Hydroinformatics

\title{
A Sliding Mode-based Predictive Strategy for Irrigation Canal Pools
}

\author{
Josenalde B. Oliveira ${ }^{12}$, Tatiana M. Pinho ${ }^{23}$, João Paulo Coelho ${ }^{24}$, José \\ Boaventura-Cunha ${ }^{23}$, and Paulo Oliveira ${ }^{23}$ \\ 1 Agricultural School of Jundiaí, Federal University of Rio Grande do Norte, UFRN, Brazil \\ josenalde@eaj.ufrn.br \\ 2 INESC TEC Technology and Science, Campus da FEUP, 4200-465 Porto, Portugal \\ 3 School of Sciences and Technology, University of Trás-os-Montes and Alto Douro, UTAD, Quinta \\ de Prados, 5000-801 Vila Real, Portugal \\ tatianap@utad.pt, jboavent@utad.pt, oliveira@utad.pt \\ 4 School of Technology and Management, Polytechnic Institute of Bragança, Campus de Sta. \\ Apolónia, 5300-253 Bragança, Portugal \\ jpcoelho@ipb.pt
}

\begin{abstract}
This paper evaluates a robust Model Predictive Controller (MPC) based on Sliding Modes (SMPC) for the downstream level control in irrigation canal pools. Its features are compared with the conventional Generalized Predictive Controller (GPC), regarding set point tracking (water level) and output disturbances (offtake discharges). Simulation results suggest feasibility of applying SMPC for gate manipulation, with suitable command signals and robustness.
\end{abstract}

\section{Introduction}

Among several functions, water transportation systems may be used for delivering water for irrigation, often from a long distance source, to the users. The main goal in canal control for agricultural purposes is to minimize the water waste when supplying water to farmers [11]. Since the off-takes are, in most cases, gravity fed, the requirement of being able to supply water is a set-point regulation of water levels (and consequently water flow rate), by using the gate opening heights (or associated discharges) as control actions [3]. This is normally accomplished by controlling the water depth at the extraction localization (downstream). This control logic transfers the offtake demands to the upstream water source, being therefore demand-oriented.

A canal pool can be divided into the uniform part (transport zone), located upstream, where water flows at approximately constant depth, and the backwater part (storage zone), where water is slow-moving or stagnant (with an approximately horizontal surface), which is caused by an obstruction (as a gate) to the flow at the downstream end. The model captures the water profile along the pool axis, the wave translation and attenuation as well as the flow acceleration, known as pool model [18, 17].

G. La Loggia, G. Freni, V. Puleo and M. De Marchis (eds.), HIC 2018 (EPiC Series in Engineering, vol. 3), pp. $1589-1597$ 
Open irrigation canals are well modelled by the Saint-Venant equations, a set of hyperbolic partial differential equations. Such canals are formed by a sequence of pools separated by gates, therefore a challenging system dynamics and control problem for distributed parameter plants, since a gate opening produces a water wave that travels through the pool which is partially reflected back in the next gate, the remainder crosses the gate and propagates to the next pool. Works such as [15] deal with the rigorous analysis of such interactions considering wave disturbances and the Boundary feedback control proposed by [6] is directly based on the Saint-Venant equations. Control oriented models usually assume a nonlinear system around equilibrium points (steady-state conditions) and typically linearized models are used for predicting the system dynamics within a Model Predictive Control (MPC) approach. Under certain assumptions, these linear models can approximate the nonlinear dynamics well. The MPC optimization for such models is easy and fast to solve and moreover global optimal solutions can be found. The most common simplified model used in practice is the Integrator Delay (ID) model [20] which considers that in low frequencies the water depth can be approximated as the integral of the flow. Its extension, the Integrator Delay with Zero (IDZ) model [16] is more general and includes a zero in the transfer function to cope with low and high frequencies. Based on the IDZ model, a suitable and simpler model for pools relating solely the downstream level (output) with the upstream gate opening (input) can be described by a second order plus dead time transfer function, as used by [10, 9, 8]. Here, the model of the first pool (Bocal) of the Aragon Imperial main canal (Ebro River, Spain), described in [10] is used as the prediction model for the MPC and the proposed Sliding Mode MPC (SMPC). MPC offers a straightforward design method to anticipate future control actions within some time horizon (control horizon), in order to track a future behavior (in some prediction horizon), predicted by an explicit model. Recent MPC approaches suggest a trend toward state space formulations which provides flexibility in representing stable, unstable, integrating and unmeasured disturbances, just as the Controller Auto-Regressive Integrated Moving-Average (CARIMA) model in the Generalized Predictive Control (GPC) $[5,1]$.

The objective function of GPC is very similar to that of Dynamic Matrix Control (DMC), with the fundamental difference of using a Diophantine equation and CARIMA model to formulate the dynamic matrix. [1] showed that GPC and Extended Predictive Control (EPC) can handle the system matrix ill-conditionality better than other MPC methods and, therefore, it still motivates the development and applications of GPC, as in [5]. Considering its wide application in environments subject to disturbances, robustness is a necessary feature and must be taken into account. An attempt to aggregate robustness into GPC by combining GPC with Sliding Mode Control (SMC) was firstly reported in [4]. For the canal problem, time varying parameters are a common issue [10] and must be properly controlled.

$\mathrm{SMC}$ is a nonlinear control scheme known to be robust to model uncertainties, disturbances and unmodeled dynamics, being quite suitable for industrial environments. Since the considerations by [23], the research on SMC theory and its applications have been of increasing interest, providing an engineering look at SMC. Key aspects were clarified, such as the chattering phenomena, both in continuous and discrete time. The key idea consists in choosing a state variables function (sliding surface) in which all trajectories must reach in finite time (reaching phase) and, once reached, can not escape, sliding to the desired final value (sliding phase). A control law is then designed to force the trajectories towards this surface (corrective action) and, moreover, to keep them thereafter (equivalent control [22]). This control law must be discontinuous or, at least, it must contain a discontinuous component.

The well succeeded melting of SMPC motivated other works and applications [7, 12, 14, 19, 13]. Following some design steps of the SMPC presented in [13], this article keeps Quadratic 


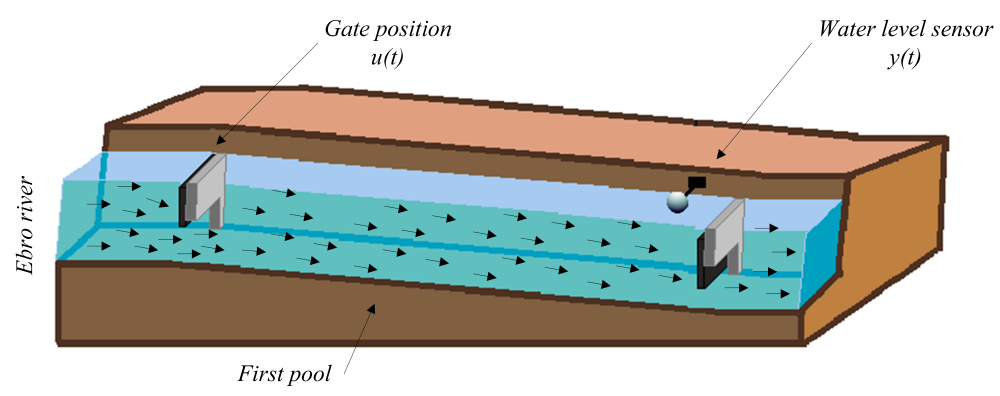

Figure 1: Schematic representation of the Ebro river irrigation canal first pool. The manipulated variable is the water level and the controlled variable is the upstream gate position [8]

Programming (QP) active-set for the optimization of the continuous component of the control law responsible for the sliding phase, and proposes a tuning rule for selecting parameters for the discontinuous component of the control law.

\section{Problem Statement}

In this article, it will be considered the problem addressed in [10] where the first pool of an irrigation canal (see Figure 1), located near the Ebro river in Spain, has been modelled by means of the following second-order transfer function:

$$
\frac{\Delta Y(s)}{\Delta U(s)}=\frac{K}{\left(T_{1} s+1\right)\left(T_{2} s+1\right)} e^{-\tau s}
$$

where $\Delta Y(s)$ and $\Delta U(s)$ denote, respectively, the downstream end water level variation and the upstream gate position variation both in centimeters. The other parameters are the static gain, $K$, the process main time constants $T_{1}$ and $T_{2}$ and the pure time delay $\tau$. The time constant $T_{1}$ is associated to the canal pool dynamics and $T_{2}$ represents the mechanical actuators dynamics. As can be expected, due to the immense inertia of the water pool, $T_{1} \gg T_{2}$. For the considered sampling time $T_{s}=60 \mathrm{~s}$, a discretized version of (1) with Zero Order Hold is:

$$
\frac{\Delta Y(z)}{\Delta U(z)}=\frac{0.0007811 z+0.0005975}{z^{2}-1.412 z+0.4465} z^{-6}
$$

and a lateral offtake discharge (output disturbance) is simulated with a first order plus dead time model, with a time constant $T_{3}=70 \mathrm{~s}$. Both continuous and discrete models $\left(T_{s}=60 \mathrm{~s}\right)$ are given below for a negative step input $\Delta f(z)$ :

$$
\frac{\Delta D(s)}{\Delta f(s)}=\frac{1}{T_{3} s+1} \Leftrightarrow \frac{\Delta D(z)}{\Delta f(z)}=\frac{0.5756}{z-0.4244}
$$

The considered irrigation pool has a trapezoidal cross section that extends down for a length of $8 \mathrm{~km}$. Using the water level measurements obtained after performing a set of step response experiments, the coefficient values of the transfer function (1) were obtained. In particular, and for a nominal operating regime, $K=0.0401, T_{1}=880.79 \mathrm{~s}, T_{2}=81.27 \mathrm{~s}$ and $\tau=360 \mathrm{~s}$. 


\section{Controller Design}

The SMPC presented here is based on a Controller Auto-Regressive Integrated Moving-Average model (CARIMA), considered linear around each operating point and described as:

$$
A\left(q^{-1}\right) \Delta y(k)=B\left(q^{-1}\right) \Delta u(k-d-1)+\xi(k),
$$

where $d$ is the delay from input to output (here considered as a multiple of the sampling time), $u$ is the input signal, $q^{-1}$ is the backward-shift operator, $\Delta: 1-q^{-1}$, and $\xi$ is the zero mean white noise. $A$ and $B$ are polynomials in $q^{-1}$ defined as:

$$
\begin{aligned}
& A\left(q^{-1}\right)=1+a_{1} q^{-1}+a_{2} q^{-2}+\ldots+a_{n a} q^{-n a}, \\
& B\left(q^{-1}\right)=b_{0}+b_{1} q^{-1}+b_{2} q^{-2}+\ldots+a_{n b} q^{-n b} .
\end{aligned}
$$

According to the SMC theory [22], the first step to design the controller is to define a sliding surface, $S(t)$, along which the process can slide to find its desired final value. Very often, $S(t)$ is chosen in such a way that represents a desired system dynamics and/or control objective. For instance, $S(t)$ could be the tracking error $e_{o}=y-w$, with $w$ being some reference signal. The problem of tracking a reference value can be reduced to keeping $S(t)$ at zero. From [12, 13], the $j$-step ahead prediction of $S(k)$ with information until the actual instant $t=k$ is given by:

$$
\hat{S}(k+j)=P_{s}\left(q^{-1}\right)(\hat{y}(k+j)-w(k+j))+Q_{s}\left(q^{-1}\right) \Delta u(k+j-1-d) .
$$

Polynomials $P_{s}\left(q^{-1}\right)$ and $Q_{s}\left(q^{-1}\right)$ have degree $n p$ and $n q$ respectively, and allow to design the desired dynamics in the sliding condition.

A common adjustment is choosing $P_{s}\left(q^{-1}\right)$ and $Q_{s}\left(q^{-1}\right)$ as:

$$
\frac{Q_{s}\left(q^{-1}\right)}{P_{s}\left(q^{-1}\right)}=\frac{(1-\alpha) q^{-1}}{1-\alpha q^{-1}}
$$

with $0<\alpha \leq 1$, since all roots of $P_{s}\left(q^{-1}\right)$ must be inside the unit circle [13]. As $\alpha \rightarrow 0$ the dynamic is faster.

The cost function aggregates two simultaneous objectives:

$$
J_{C}=\sum_{j=N_{1}}^{N_{y}}[\hat{S}(k+j)]^{2}+\sum_{j=1}^{N_{u}} \lambda[\Delta u(k+j-1)]^{2}
$$

where $\lambda$ is set constant and $N_{1}-N_{y}$ is the period of time in which one desires the output tracks the reference signal and $N_{u}$ is the control horizon. For these parameters, [13] suggested some intuitive relations, which can be used as initial values. Other online tuning strategies for these specific parameters are available in literature [2].

Likewise GPC, to minimize (9) the output prediction within the interval $j=\left[N_{1}, N_{y}\right]$ is formed by two parts:

$$
\hat{y}(k+j)=y_{f}(k+j)+y_{l}(k+j),
$$

where $y_{f}$ is the forced response (considering the initial conditions null but subject to future control actions) and $y_{l}$ is the free response (natural system response from the initial conditions 
with no future control actions). The forced response $y_{f}$ may be calculated from the step response of the parametric model ( $H$ matrix described in [19]) (4):

$$
\mathbf{y}_{f}=H \cdot \Delta \mathbf{u}
$$

where $\mathbf{y}_{f}=\left[y_{f}(1), \cdots y_{f}\left(N_{u}\right)\right]$ and $\Delta \mathbf{u}_{c}=\left[\Delta u_{c}(1), \cdots, \Delta u_{c}\left(N_{u}\right)\right]$.

\subsection{Prediction of the Sliding Surface}

SMPC control law, $\Delta u_{S M P C}(k)$, is the combination of two additive parts: a continuous part $\Delta u_{c}(k)=\Delta u(k)$ developed like a GPC by the minimization of (9) using quadratic programming (QP), which is responsible for keeping the process variable on the reference value, and a discontinuous part $\Delta u_{d}(k)$ to be detailed further, responsible for guiding the system to the sliding surface. To calculate $\Delta u_{c}(k),(10)$ is substituted into (7) and, after putting it into matrix form, one has:

$$
\hat{\mathbf{S}}=\left(P_{s} H+Q_{s}\right) \Delta \mathbf{u}_{c}+P_{s}\left(\mathbf{y}_{l}-\mathbf{w}\right),
$$

with $P_{s}$ a square matrix of dimension $\left(N_{y}-N_{1}-1\right) \times\left(N_{y}-N_{1}-1\right), Q_{s}$ a matrix of size $\left(N_{y}-\right.$ $\left.N_{1}-1\right) \times N_{u}$ composed after $(9), \hat{\mathbf{S}}=\left[\hat{S}(1), \cdots, \hat{S}\left(N_{u}\right)\right]$. Additionally, w $=\left[w(1), \cdots w\left(N_{u}\right)\right]$, $p_{s 0}=1, p_{s 1}=-\alpha, q_{s 0}=0, q_{s 1}=1-\alpha, n p=1$ and $n q=1$.

Substituting (11) into (9), the cost function becomes:

$$
J_{C}=\frac{1}{2} \Delta \mathbf{u}_{c}^{T} G \Delta \mathbf{u}_{c}+\Delta \mathbf{u}_{c}^{T} b+f_{0}
$$

with $G=2\left(\lambda_{1} Z^{T} Z+\lambda_{2} I\right), Z=P_{s} H+Q_{s}, f_{0}=\lambda_{1}\left(P_{s}\left(\mathbf{y}_{l}-\mathbf{w}\right)\right)^{T}\left(P_{s}\left(\mathbf{y}_{l}-\mathbf{w}\right)\right)$ and $b=$ $2 \lambda_{1} Z^{T} P_{s}\left(\mathbf{y}_{l}-\mathbf{w}\right)$.

A necessary and sufficient condition for discrete time reaching motion is $|\hat{S}(k+1)| \leq \hat{S}(k)$ [13] and a control law satisfying this condition does guarantee that all trajectories will enter and remain within at least a non-increasing domain. In order to guarantee the reaching condition of (12), a constraint is added into the optimization problem of (13), namely:

$$
-\hat{\mathbf{S}} \cdot \mathbf{1}+P_{s}\left(\mathbf{y}_{l}-\mathbf{w}\right) \leq\left(P_{s} H+Q_{s}\right) \Delta \mathbf{u}_{c} \leq \hat{\mathbf{S}} \cdot \mathbf{1}-P_{s}\left(\mathbf{y}_{l}-\mathbf{w}\right)
$$

where $\mathbf{1}_{N u \times 1}$ is a vector whose entries are ones.

\subsection{Discontinuous Control Signal Component}

The discontinuous part $\Delta u_{d}$ is given here by its smooth version, replacing the sign function by a hyperbolic tangent function:

$$
\Delta u_{d}(k+j)=K_{d} \cdot \tanh (\hat{S}(k+j)),
$$

where $K_{d}$ is a gain responsible for the velocity of the reaching mode but also increases chattering. To improve both tracking and disturbance rejection, this work uses an adaptive $K_{d}$, similar to the one proposed in [21], defined as:

$$
K_{d}=(1-\mu) K_{d} R+\mu K_{d} S S
$$

with

$$
\mu=e^{\frac{-e_{0}^{2}}{L}}, \quad \mu_{0}<\mu \leq 1
$$


where $e_{0}$ represents the tracking error, $\mu_{0}$ the lower bound for $\mu, L=0.2$, and $K_{d} R$ and $K_{d} S S$ are, respectively, the rising and steady-state components of $K_{d}$. In this case, $K_{d} R=0.28$ and $K_{d} S S=6.52$.

Therefore, the complete control signal increment for the SMPC at $t=k$ is:

$$
\Delta u_{S M P C}(k)=\Delta u_{c}(k)+\Delta u_{d}(k) .
$$

It is noteworthy that at each instant $k$, although $N_{u}$ components are calculated for $\Delta u_{c}$ and $\Delta u_{d}$, only the first component of each $\left(u_{c}(k), u_{d}(k)\right)$ is considered, neglecting the other components, obeying the receding horizon principle. Then, the actual control signal sent to the process is given by:

$$
u_{S M P C}(k)=u_{S M P C}(k-1)+u_{c}(k)+u_{d}(k) .
$$

\section{Simulation Results and Discussion}

This section deals with the obtained results concerning the application of SMPC to the problem of water level regulation in the first pool of the Ebro river irrigation canal, and its performance compared with a MPC. All simulations represent a total time of $30,000 s$, with a fixed step size of $60 \mathrm{~s}$. An offtake discharge reduces by $0.3 \mathrm{~m}$ the downstream water level, introduced at $t=20,000 \mathrm{~s}$. The input constraints are $u_{S M P C} \in[0 \mathrm{~cm}, 100 \mathrm{~cm}], \Delta u_{S M P C} \in[-25 \mathrm{~cm}, 25 \mathrm{~cm}]$. The canal has a variable depth between $3.7 \mathrm{~m}$ and $3.1 \mathrm{~m}$. Besides, $\lambda_{1}=\lambda_{2}=1, N_{y}=100, N_{u}=20, \alpha=0.3$ and the MPC was equally tuned with these parameters.

The results are compared in terms of overshoot, rise time, settling time, ITAE, ISE, IAE, total control signal and control signal standard deviation, which are presented in Table 1. All these indexes must be as small as possible, but an usual trade-off between tracking and control effort is expected, and therefore it must be addressed according to the problem, constraints and performance requirements. Figure 2 shows the system output for the MPC and SMPC for a unitary reference. Also, a steady-state setpoint error tolerance of $5 \%$, represented by the red lines, is considered acceptable.

\begin{tabular}{lrr}
\hline Index & MPC & SMPC \\
\hline Overshoot (\%) & 0.05 & 0.24 \\
Rise time (s) & 3110.4 & 2879.8 \\
Settling time (s) & 5293.3 & 5018.5 \\
ITAE & 5677.8 & 5337.4 \\
ISE & 39.46 & 33.50 \\
IAE & 60.16 & 53.50 \\
Total control signal & 13005 & 13208 \\
Control signal standard deviation & 6.11 & 5.59 \\
\hline
\end{tabular}

Table 1: MPC and SMPC performance indexes

By analysing the information provided in Table 1 and Figure 2, the SMPC shows a faster tracking and disturbance rejection ability, when compared with MPC. Although the SMPC presents a slightly higher overshoot, this value is negligible within the considered steady-state error tolerance. The main advantage of SMPC is its ability to increase the overall system response, which can be observed in Figure 2. This increased dynamic performance is represented by lower values in terms of rise time and settling time. Also, the SMPC has a lower control effort 


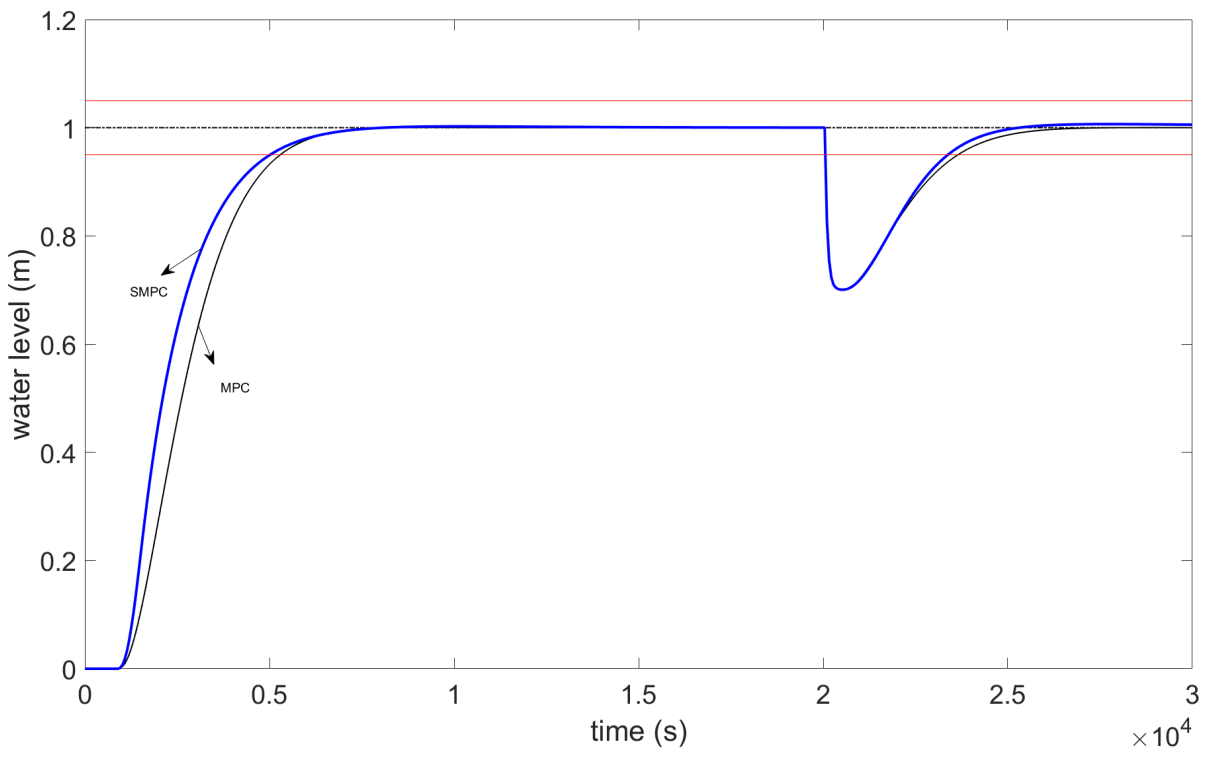

Figure 2: Water level using MPC and SMPC control strategies

observed in the lower value of the control signal standard deviation. The overall performance indexes for SMPC are smaller, showing a feasible application without significant control signal increase, mainly for discharge actions.

\section{Conclusions}

The problem of water level regulation, in irrigation canal pools, represents a major challenge for control systems design. This is due to the large dynamic variations that these systems exhibit during their operating conditions. To overcome this fact, robust controllers should be applied. In this work, a SMPC approach was used to regulate the water level in an irrigation canal pool. Simulations, using a mathematical model of the system, have been elaborated in order to evaluate the performance of the proposed control methodology. The obtained results were compared to the ones provided using conventional MPC. The simulation outcomes indicate that SMPC provides better results regarding the defined set of performance indexes. Thus, SMPC presents promising features concerning the disturbance rejection and set-point tracking while maintaining the control signals within its dynamic operating range. Nevertheless, this method can be further improved by defining the $K_{d}$ value through optimization algorithms for online operation. Moreover, this control method must be further validated by applying it in a real or scaled physical process.

\section{References}

[1] M. Abu-Ayyad and R. Dubay. Real-time comparison of a number of predictive controllers. ISA Transactions, 46(3):411-418, jun 2007. 
[2] Ashraf Al-Ghazzawi, Emad Ali, Adnan Nouh, and Evanghelos Zafiriou. On-line tuning strategy for model predictive controllers. Journal of Process Control, 11(3):265-284, jun 2001.

[3] Lihui Cen, Ziqiang Wu, Xiaofang Chen, Yanggui Zou, and Shaohui Zhang. On modeling and constrained model predictive control of open irrigation canals. Journal of Control Science and Engineering, 2017:1-10, 2017.

[4] M.Letizia Corradini and Giuseppe Orlando. A VSC algorithm based on generalized predictive control. Automatica, 33(5):927-932, may 1997.

[5] Mark L. Darby and Michael Nikolaou. MPC: Current practice and challenges. Control Engineering Practice, 20(4):328-342, apr 2012.

[6] J. de Halleux, C. Prieur, J.-M. Coron, B. Andréa-Novel, and G. Bastin. Boundary feedback control in networks of open channels. Automatica, 39(8):1365-1376, aug 2003.

[7] Mercedes Pérez de la Parte, Oscar Cammacho, and Eduardo F. Comacho. Development of a GPC-based sliding mode controller. ISA Transactions, 41(1):19-30, jan 2002.

[8] Josenalde Barbosa de Oliveira, Tatiana M. Pinho, João Paulo Coelho, José Boaventura-Cunha, and Paulo Moura Oliveira. Optimized fractional order sliding mode controller for water level in irrigation canal pool. IFAC-PapersOnLine, 50(1):7663-7668, jul 2017.

[9] Jorge Domingues, Duarte Valerio, and José Sá da Costa. Rule-based fractional control of an irrigation canal. In 2009 35th Annual Conference of IEEE Industrial Electronics, pages 1-6. IEEE, nov 2009.

[10] V. Feliu-Batlle, R. Rivas-Perez, F.J. Castillo-Garcia, L. Sanchez-Rodriguez, and A. Linarez-Saez. Robust fractional order controller for irrigation main canal pools with time-varying dynamical parameters. Computers and Electronics in Agriculture, 76(2):205-217, may 2011.

[11] João Figueiredo, Miguel Ayala Botto, and Manuel Rijo. SCADA system with predictive controller applied to irrigation canals. Control Engineering Practice, 21(6):870-886, jun 2013.

[12] Winston García-Gabín and Eduardo F. Camacho. Sliding mode model based predictive control for non minimum phase systems. In European Control Conference (ECC), 2003.

[13] Winston García-Gabín, Darine Zambrano, and Eduardo F. Camacho. Sliding mode predictive control of a solar air conditioning plant. Control Engineering Practice, 17(6):652-663, jun 2009.

[14] Winston García-Gabín, Darine Zambrano, and E.F. Camacho. Sliding mode predictive control for chemical process with time delay. IFAC Proceedings Volumes, 38(1):627-632, 2005.

[15] J. M. Igreja. Rigorous numerical method for irrigation canal system dynamics and control. In 2015 Proceedings of the Conference on Control and its Applications, pages 93-100. Society for Industrial and Applied Mathematics, jul 2015.

[16] Xavier Litrico and Vincent Fromion. Analytical approximation of open-channel flow for controller design. Applied Mathematical Modelling, 28(7):677-695, jul 2004.

[17] João Nabais and Miguel Ayala Botto. Linear model for canal pools. In ICINCO 2011 - Proceedings of the 8th International Conference on Informatics in Control, Automation and Robotics, volume 1, pages 306-313, 2011.

[18] João Miguel Lemos Chasqueira Nabais, José Duarte, Miguel Ayala Botto, and Manuel Rijo. Flexible framework for modeling water conveyance networks. In SIMULTECH 2011 - Proceedings of 1st International Conference on Simulation and Modeling Methodologies, Technologies and Applications, pages 142-147, July 2011.

[19] J. B. Oliveira, J. Boaventura-Cunha, and P. B. Moura Oliveira. A feasibility study of sliding mode predictive control for greenhouses. Optimal Control Applications and Methods, 37(4):730-748, jul 2016.

[20] J. Schuurmans. Control of Water Levels in Open Channels. PhD thesis, Delft University of Technology, Netherlands, 1997.

[21] Leonardo R.L. Teixeira, Josenalde B. Oliveira, and Aldayr D. Araujo. Smooth indirect adaptive sliding mode control. International Journal of Robust and Nonlinear Control, 25(6):775-790, nov 
2015.

[22] Vadim I. Utkin. Sliding Modes in Control and Optimization. Springer Berlin Heidelberg, 1992.

[23] K.D. Young, V.I. Utkin, and U. Ozguner. A control engineer's guide to sliding mode control. IEEE Transactions on Control Systems Technology, 7(3):328-342, may 1999. 